\title{
Comparison of intravaginal and oral metronidazole in patients with bacterial vaginosis
}

\author{
Sang Won Han, Jae Eun Chung*
}

Department of Obstetrics and Gynecology, National Health Insurance Service Ilsan Hospital, Goyang, South Korea

Received: 04 August 2017

Accepted: 01 September 2017

\section{*Correspondence: \\ Dr. Jae Eun Chung, \\ E-mail: jiupark@naver.com}

Copyright: (C) the author(s), publisher and licensee Medip Academy. This is an open-access article distributed under the terms of the Creative Commons Attribution Non-Commercial License, which permits unrestricted non-commercial use, distribution, and reproduction in any medium, provided the original work is properly cited.

\begin{abstract}
Background: Metronidazole is considered an effective treatment for bacterial vaginosis. Only oral preparations were available until 2014, but an intravaginal gel preparation recently became commercially available in South Korea. In this report, the efficacy and safety of metronidazole intravaginal gel application was compared to that of the conventional oral metronidazole preparation.

Methods: Patients who were treated with either intravaginal metronidazole or oral metronidazole after diagnosis of bacterial vaginosis were included in this study. Patient characteristics, mode of treatment, recurrence rate, and complications including gastro-intestinal discomfort and concomitant vulvovaginal candidiasis were analyzed.

Results: There were no differences in patient characteristics, except the mean age was older in the intravaginal group. Treatment outcomes were similar in both groups. In the oral metronidazole group, $6.3 \%$ of patients reported ongoing symptoms of bacterial vaginosis within one month of treatment and required a refill, while $7.3 \%$ of the intravaginal group required a refill. Significantly more patients on oral metronidazole treatment complained about gastro-intestinal discomfort including nausea (13.4\%) and diarrhea (9.4\%). Patients who took intravaginal metronidazole complained about increased watery vaginal discharge $(26.8 \%)$. The comparatively high cost of metronidazole intravaginal gel was another factor that could affect patient access to this treatment versus the oral preparation. The incidence of vulvovaginal candidiasis was similar between groups (oral $3.9 \%$, intravaginal $4.9 \%$ ).

Conclusions: Intravaginal metronidazole usage can be considered as an effective alternative treatment for bacterial vaginosis in patients with gastro-intestinal complications. However, the increased incidence of watery vaginal discharge and high cost remain obstacles to widespread use of the intravaginal preparation.
\end{abstract}

Keywords: Bacterial vaginosis, Metronidazole ointment, Metronidazole gel, Vaginitis

\section{INTRODUCTION}

Bacterial vaginosis is one of the most common disease entities observed in gynecology, with a prevalence rate of approximately $30 \%$ in the female population. ${ }^{1}$ Increased vaginal discharge with foul odor are the typical manifestations of bacterial vaginitis. These uncomfortable symptoms not only interfere with everyday activities, but might also result in preterm labor in pregnant patients, or may increase the incidence of postsurgical infections. ${ }^{2}$ Instead of a single pathogen causing these inflammatory changes, multiple pathogens are thought to be involved. Therefore, keeping multiple pathogens under control as well as maintaining normal vaginal flora should be the goals of bacterial vaginosis prevention and treatment. ${ }^{3}$ Although multiple pathogens are involved in the occurrence of bacterial vaginosis, most cases are well controlled by metronidazole antibiotics. In 2002, the Centers of Disease Control recommended both oral and intravaginal metronidazole application for the treatment of bacterial vaginosis. ${ }^{4}$ Oral intake of metronidazole has been reported to have 
complications such as nausea, vomiting, diarrhea, change of taste, and gas distention. ${ }^{5}$ These complications often occur in patients with gastritis or those who take multiple medications together. Intravaginal application of metronidazole could be considered in patients who experience gastro-intestinal discomfort while taking oral metronidazole. In South Korea, an intravaginal metronidazole preparation became available in 2014. The efficacy and side effects of oral and intravaginal metronidazole treatment were compared in this study.

\section{METHODS}

Medical records of patients treated under the diagnosis of bacterial vaginosis from July 2014 to March 2015 were reviewed. Either an oral metronidazole preparation (metronidazole $250 \mathrm{mg}$, Flasinyl tablet, CJ Pharmaceutical) or intravaginal metronidazole gel (MEROGEL 10 gm, BC World Medical) were prescribed. Pregnant patients and those with concomitant infection with Chlamydia trachomatis, trichomoniasis, or candidiasis were excluded from the study. Those with intrauterine devices were also excluded due to the possibility of actinomycosis infection.

Both the oral and intravaginal preparations were prescribed for 5 days. Patients were followed up at an outpatient clinic after 1 week. Treatment failure was defined as when the patient's symptoms did not improve after 5 days of treatment. Recurrence of bacterial vaginosis was defined as an initial improvement in symptoms followed by recurrent bacterial vaginosis. Age, menopause status, diabetes mellitus, oral contraceptive use, side effects, recurrence, treatment failure, and preferred modality of metronidazole data were retrieved from the medical records of each patient.

The chi-squared test was used for comparative analysis of categorical variables in order to determine independent risk factors. Statistical significance was defined as $\mathrm{P}<$ 0.05. SPSS 17.0 software (SPSS, Chicago, IL, USA) was used.

\section{RESULTS}

Table 1: Patient's characteristics.

\begin{tabular}{|llll|}
\hline & Oral metronidazole (127) & Intravaginal metronidazole (41) & P value \\
\hline $\begin{array}{l}\text { Mean age (SD)* } \\
\begin{array}{l}\text { Previous history of } \\
\text { bacterial vaginosis (\%) }\end{array}\end{array}$ & $33.5(10.2)$ & $42.3(13.2)$ & 0.001 \\
\hline $\begin{array}{l}\text { Previous history of vulvovaginal } \\
\text { candidiasis (\%) }\end{array}$ & 5.3 & 6.1 & 0.341 \\
\hline Diabetes (\%)* & 2.4 & 3.2 & 0.275 \\
\hline Contraceptives (\%)* & 2.0 & 6.8 & 0.001 \\
\hline Menopause (\%)* & 7.9 & 2.1 & 0.001 \\
\hline
\end{tabular}

${ }^{*} \mathrm{p}<0.05$ Pearson's chi-square test

Table 2: Treatment outcome of metronidazole.

\begin{tabular}{|llll|} 
& Oral metronidazole (127) & Intravaginal metronidazole (41) & P value \\
\hline Treatment failure & $8(6.3 \%)$ & $3(7.3 \%)$ & 0.351 \\
\hline Recurrent Bacterial vaginosis & $25(19.7 \%)$ & $9(21.9 \%)$ & 0.243 \\
\hline
\end{tabular}

*p<0.05 Pearson's chi-square test

Table 3. Complications.

\begin{tabular}{|lll|}
\hline Nausea & Oral metronidazole (127) & Intravaginal metronidazole (41) \\
\hline Bloating sensation & $17(13.4 \%)$ & $0(0 \%)$ \\
\hline Diarrhea & $12(9.4 \%)$ & $0(0 \%)$ \\
\hline Unpleasant taste & $9(7.1 \%)$ & $0(0 \%)$ \\
\hline Increased vaginal discharge & $9(7.1 \%)$ & $0(0 \%)$ \\
\hline Vulvovaginal candidiasis & $0(0 \%)$ & $11(26.8 \%)$ \\
\hline Cost problem & $5(3.9 \%)$ & $3(4.9 \%)$ \\
\hline
\end{tabular}

There were 127 oral and 41 intravaginal metronidazole patients. Mean age was older in the intravaginal group
(33.5 \pm 10.2 vs. $42.3 \pm 13.2$ years). More patients were menopausal in the intravaginal group. There were no 
differences in bacterial vaginosis and candidiasis history. In total, $6.8 \%$ of the intravaginal group had diabetes mellitus, and $7.9 \%$ were taking oral contraceptives in the oral group (Table 1).

Of the 127 oral metronidazole patients, 8 experienced treatment failure $(6.3 \%)$. Of the 41 intravaginal metronidazole patients, 3 experienced treatment failure $(7.3 \%)$. This was not significantly different between the 2 groups. Concerning the recurrence of bacterial vaginosis, no statistically significant difference was found as well (Table 2).

Patients taking oral metronidazole reported various gastro-intestinal symptoms including nausea, bloating, diarrhea, and unpleasant taste. Patients taking intravaginal metronidazole experienced increased vaginal discharge and vulvovaginal candidiasis.

The higher cost of the intravaginal preparation was one of the factors that affected patient preference for the oral preparation (Table 3).

\section{DISCUSSION}

One of the most common disease entities encountered in outpatient gynecology is bacterial vaginosis. ${ }^{1}$ With the onset of female hormone production, lactobacilli, the normal flora in the vagina, transform glycogen to lactic acid. The production of lactic acid maintains the vaginal $\mathrm{pH}$ between 3.8 and 4.2. This acidic environment is hostile for anaerobes. The resulting normal vaginal flora consists of $95 \%$ lactobacilli and $5 \%$ anaerobes. ${ }^{6}$ Bacterial vaginosis is defined as the loss of ecological balance of lactobacilli and anaerobes, resulting in overgrowth of anaerobes. $^{2,4}$

The representative signs of bacterial vaginosis are a foul odor resembling that of decaying fish, which is caused by anaerobe production of amine-like materials, and/or milky/yellowish vaginal discharge. The foul odor worsens during menstruation and intercourse because the alkalinity of blood and semen increases intravaginal $\mathrm{pH}$, rendering the intravaginal environment favorable to the growth of anaerobes. Anaerobes also secrete enzymes that decompose vaginal mucosa. This exfoliated decomposed vaginal mucosa is observed as increased vaginal discharge by the patient. ${ }^{7}$

Gardnerella vaginalis, Prevotella species, Mobiluncus species, and Mycoplasma hominis are examples of anaerobes found in the vagina. Newly-found anaerobes such Atopobium vaginalis have also been added to this list of anaerobes naturally occurring in the vagina. ${ }^{8}$ Fortunately, most of these anaerobes can be controlled by metronidazole. ${ }^{2,4}$

Important pathogens that should be distinguished from bacterial vaginosis-causing anaerobes include Chlamydia trachomatis and trichomoniasis. These pathogens are included in the category of sexually transmitted diseases and have the ability to migrate into the upper reproductive organs such as the uterus and adnexa. Infection of the upper reproductive organs might result in chronic pelvic inflammatory disease, infertility, and FitzHugh-Curtis syndrome. ${ }^{9}$ Instead of metronidazole, doxycycline or azithromycin should be prescribed because these pathogens do not respond to metronidazole. ${ }^{10,11}$ Patients with Chlamydia trachomatis, trichomoniasis, or actinomycosis were excluded from this study.

In the intravaginal metronidazole group, the mean age was older and the percentage of menopausal patients was higher. Due to multiple accompanying medical conditions such as hypertension, hypercholesterolemia, and DM that need to be controlled with daily medication, many older patients preferred the intravaginal preparation to alleviate the burden of taking more medications per os (Table 1).

Treatment failure and recurrence of bacterial vaginosis might result from incomplete restoration of the normal vaginal flora. Repetitive and excessive usage of vaginal detergents and douching are considered the main culprits of the failure to restore normal vaginal flora. There were no differences in treatment failure and recurrence between the oral and intravaginal metronidazole groups (Table 2).

Definite differences were noted in the complications accompanying the two different metronidazole preparations. In the oral group, most side effects were gastro-intestinal, whereas in the intravaginal group increased vaginal discharge was the chief complaint. Vaginal discharge occurring after intravaginal metronidazole application was not yellowish nor was it accompanied by a foul odor, which are the primary characteristics of bacterial vaginosis. Discharge is thought to consist of non-absorbed metronidazole gel components. Although this discharge was not associated with treatment failure or recurrence, the presence of the discharge itself may have resulted in contact vaginitis due to continuous usage of sanitary napkins. The cost of the intravaginal preparation was approximately 4-fold that of the oral preparation. Candidiasis associated with antibiotic usage has been reported previously. ${ }^{12}$ In this study, $3.9 \%$ of the oral and $4.9 \%$ of the intravaginal group experienced vulvovaginal candidiasis after metronidazole treatment; this difference was not statistically significant (Table 3 ).

Attempts to restore normal vaginal flora by supplementing lactobacilli in the vaginal cavity have not been effective. The ability of lactobacilli to produce $\mathrm{H} 2 \mathrm{O} 2$ while adhering to the vaginal wall is a key component of maintaining vaginal $\mathrm{pH}$ under $4.2 .^{13}$ The lack of adherence to the vaginal wall and minimal production of $\mathrm{H} 2 \mathrm{O} 2$ by marketed probiotics hinders their usage as a treatment modality for bacterial vaginosis. ${ }^{14}$ 
Bacterial vaginosis causes discomfort in women due to foul odor and increased vaginal discharge, and could lead to serious consequences such as preterm labor or premature rupture of membranes in pregnancy. ${ }^{15}$ Therefore, appropriate treatment with antibiotics to suppress the activity of multiple pathogens as well as to restore normal vaginal flora is important. The results of this study demonstrate that there are no differences in the efficacy of the two different metronidazole modalities, but the associated complications varied considerably. Further research is warranted on the development of probiotics that might restore normal vaginal flora.

Funding: No funding sources

Conflict of interest: None declared

Ethical approval: The study was approved by the Institutional Ethics Committee

\section{REFERENCES}

1. Allsworth H, Peipert J. Prevalence of bacterial vaginosis: 2001-2004 National Health and Nutrition Examination Survey data. Obstet Gynecol. 2007;109:114-20.

2. ACOG practice bulletin. Clinical management guidelines for obstetrician-gynecologists, Number 72, May 2006: Vaginitis. Obstet Gynecol. 2006;107:1195-1206.

3. Faro S, Martens M, Maccato M. Vaginal flora and Pelvic Inflammatory Disease. Am J Obstet Gynecol. 1993;169:470-4.

4. Centers for Disease Control and Prevention. Sexually transmitted diseases treatment guidelines 2002. MMWR;51(RR-6):1-80.

5. Swedberg J, Steiner JF, Deiss F. Comparison of single-dose versus one-week course of metronidazole for symptomatic bacterial vaginosis. JAMA. 1985;1254:1046-9.

6. Brabin L. Interactions of the female hormonal environment, susceptibility to viral infections, and disease progression. AIDS Patient Care STDs. 2002;15:211-21.

7. Klebanoff M, Schwebke J, Zhang J, Nansel T. Vulvovaginal symptoms in women with bacterial vaginosis. Obstet Gynecol. 2004;104:267-72.

8. Polatti F. Bacterial Vaginosis, Atopobium vaginae and Nifuratel. Current clinical pharmacol. 2012;7:3640 .

9. Haggerty C, Gottilieb S. Risk of sequele after chlamydia trachomatis genital infection in women. J Infect Dis. 2010;201(suppl 2):S134-55.

10. Centers for Disease Control and Prevention. Sexually transmitted diseases treatment guidelines 2006. MMWR;55:1-94.

11. Simpson T, Merchant J, Grimley DM, Kim O. Vaginal douching among adolescents and young women: more challenges than progress. J Pediatr Adolesc Gynecol. 204;17:249-55.

12. Eckert L, Hawes S, Stevens C. Vulvovaginal candidiasis: Clinical manifestations, risk factors, management algorithm. Obstet Gynecol. 1998;92:757-65.

13. Vallor A, Antonia M, Hawes S, Hillier S. Factors associated with acquisition of, or persistent colonization by, vaginal lactobacilli: Role of hydrogen peroxide production. $J$ Infec Dis 2001;184:1431-6.

14. Hughes VL, Hillier SL. Microbiologic characteristics of Lactobacillus products used for colonization of the vagina. Obstet Gynecol. 1990;75244-248.

15. Donders G, Van Calsteren K, Bellen G. Predictive value for preterm birth on abnormal vaginal flora, bacterial vaginosis and aerobic vaginitis during the first trimester of pregnancy. BJOG. 2009;116:131524.

Cite this article as: Han SW, Chung JE. Comparison of intravaginal and oral metronidazole in patients with bacterial vaginosis. Int J Reprod Contracept Obstet Gynecol 2017;6:4213-6. 